\title{
Signal Processing in Advanced Nondestructive Materials Inspection
}

\author{
João Manuel R. S. Tavares ${ }^{1}$ and João Marcos A. Rebello ${ }^{2}$ \\ ${ }^{1}$ Department of Mechanical Engineering, Faculty of Engineering, University of Porto, Rua Dr. Roberto Frias, 4200-465 Porto, Portugal \\ ${ }^{2}$ Department of Metallurgy and Materials, Faculty of Engineering, Federal University of Rio de Janeiro, Technological Center, Room \\ F-210, Ilha do Fundão, RJ, Brazil
}

Correspondence should be addressed to João Manuel R. S. Tavares, tavares@fe.up.pt

Received 31 October 2010; Accepted 31 October 2010

Copyright (C) 2010 J. M. R. S. Tavares and J. M. A. Rebello. This is an open access article distributed under the Creative Commons Attribution License, which permits unrestricted use, distribution, and reproduction in any medium, provided the original work is properly cited.

Over the last decades, a large number of new and improved nondestructive testing (NDT) techniques have been successfully developed. For example, NDT techniques have proven to be especially effective and reliable in crack detection and sizing due to more stringent design requirements of the current structures and equipments. Nuclear power plants, offshore platforms for deep water oil extraction, and aircraft and aerospace engines are some promising examples to which the NDT inspection techniques can be applied aiming to assure that defect detection and characterization is successfully achieved.

One of the major problems faced by NDT techniques is the difficulty on the efficient and truthful processing and analysis of the acquired signals and images. Examples can be effortlessly found in situations in which the defects either are so closely spaced that it becomes extremely difficult to detach them or are located in environments that introduce corrupted data in the acquired signals.

Digital signal processing concepts have been successfully applied to NDT for detecting, conditioning, and automatically classifying a large variety of defects as well as in the characterization of materials. Actually, digital signal processing concepts allow, for example, the improvement of the time resolution and signal-to-noise ratio and also the exposing of some details that would be hardly ascertained from the raw signals. Additionally, pattern recognition strategies are very often employed in NDT for automatically classifying the findings.

Furthermore, NDT characterization has been capable of detecting alterations in material properties. In this case, some physical properties, as frequency-dependent sound velocity and attenuation, are used. However, the practical difficulty of extracting the information needed also demands the use of proficient methods of signal processing.

This issue of the EURASIP Journal on Advances in Signal Processing constitutes the first special issue related with signal processing in advanced nondestructive materials inspection, and covers numerous outstanding topics such as pattern recognition using fractal analyses applied to several specific NDT techniques. In the same way, a classifier based on independent component analysis mixture modelling using ultrasonic was used in the characterization of materials of archaeological interest, a modified Hopfield neural network with a novel cost function was presented for detecting the boundaries of wood defects, and a neural network-based machine learning approach was employed for locating acoustic emission signals.

Additionally, the characterization of materials deserved special attention from several papers: the short time Fourier transform of ground penetration radar waveforms was used to determine concrete hydration properties; attenuation analysis of Lamb waves used the Chirplet transform in aluminum plates; piezoresistive properties of engineered cementitious composites were used as their own sensors to quantify their resistivity-strain relationship; a geometrical estimator of the time-frequency ultrasonic response of some dispersive materials was adopted. The technique of ground penetration radar was also focused on in a paper where a fast algorithm for 3D migration was developed, aiming to investigate its application in a medium that is vertically 
heterogeneous, as is the case of layered structures such as walls, floors, and pavements.

In respect to image analysis, computed tomography was used to determine fiber-length distribution in fiberreinforced polymer components, and corrosion in carbon steel storage tanks was evaluated by using the Fisher linear discriminant analysis of visual digital images. Furthermore, grain growth direction in electrodeposited copper foil was measured by means of cyclic biaxial stress and image treatment.

Moreover, defect detection and sizing in pipeline welds were performed by digital radiography, and cracks in rivet were studied by the magneto optic technique. A novel method of inspection, which employs hybrid magnetic optical strain-gage techniques, was used for the automatic monitoring of armor layers of flexible risers in the oil and gas industry.

For this special issue, 22 works, from 10 countries (Algeria, Brazil, China, France, Germany, Italy, Japan, Slovenia, Spain, and the USA) were submitted. Then, 15 works were accepted for publication after being thoroughly reviewed by international experts on NDT.

\section{Acknowledgments}

The guest editors would like to express their deep gratitude to the Editor-in-Chief and Associate Editors of EURASIP Journal on Advances in Signal Processing for this opportunity, to all authors who shared their excellent works with us, and to all members of the Scientific Committee of this special issue, helped us in the review process.

João Manuel R. S. Tavares João Marcos A. Rebello 